Review

\title{
Entanglement Entropy of AdS Black Holes
}

\author{
Mariano Cadoni * and Maurizio Melis \\ Dipartimento di Fisica, Università di Cagliari, and INFN sezione di Cagliari, Cittadella Universitaria \\ 09042 Monserrato, Italy; E-Mail: maurizio.melis@ca.infn.it
}

* Author to whom correspondence should be addressed; E-Mail: mariano.cadoni@ca.infn.it.

Received: 25 September 2010 / Accepted: 13 October 2010 / Published: 2 November 2010

\begin{abstract}
We review recent progress in understanding the entanglement entropy of gravitational configurations for anti-de Sitter gravity in two and three spacetime dimensions using the AdS/CFT correspondence. We derive simple expressions for the entanglement entropy of two- and three-dimensional black holes. In both cases, the leading term of the entanglement entropy in the large black hole mass expansion reproduces exactly the Bekenstein-Hawking entropy, whereas the subleading term behaves logarithmically. In particular, for the BTZ black hole the leading term of the entanglement entropy can be obtained from the large temperature expansion of the partition function of a broad class of 2D CFTs on the torus.
\end{abstract}

Keywords: black holes; entanglement entropy; holographic principle

\section{Introduction}

Entanglement is one of the most basic features of quantum mechanics. Historically, it has generated a long debate about the nondeterministic character of quantum mechanics. More recently, it has played a crucial role for the development of new areas of research, such as quantum information and quantum computing. Entanglement is a characterization of the spatial correlations between parts of a quantum system. It is measured by the entanglement entropy (EE), which is the von Neumann entropy arising when the degrees of freedom in an unobservable part of the system are traced over.

In recent years the notion of $\mathrm{EE}$ has been widely used for investigating general features of quantum field theory (QFT) and quantum phases of matter (e.g., spin chains and quantum liquids, [1-4]). Moreover, in the context of the anti-de Sitter/conformal field theory (AdS/CFT) correspondence, an interesting geometric interpretation has been proposed for the EE of CFTs with gravitational duals [5-7]. 
As first recognized by 't Hooft [8-16], EE could also play a key role for understanding the microscopic origin of the black hole entropy and for solving the information puzzle in black hole physics. A quantum state in a black hole geometry is divided by the horizon into two disconnected parts, and an external observer has to trace over the part of the state in the black hole interior. Another strong hint pointing to a fundamental relationship between entanglement and Bekenstein-Hawking (BH) entropy is that both quantities scale as the area of the boundary.

Unfortunately, any attempt to explain the BH entropy as due to quantum entanglement is plagued by both conceptual and technical difficulties.

First, the usual statistical interpretation of the BH entropy-aiming to explain the black hole entropy in terms of microstates - is conceptually very different from the EE, which measures the observer's lack of information about the quantum state of the system in an inaccessible region of spacetime. The problem becomes even more involved going beyond the semiclassical approximation. In fact, in the usual Euclidean quantum gravity formulation the metric, except its boundary value, cannot be fixed a priori (see e.g., [17]), whereas the usual flat-space notion of EE requires to fix lengths in bulk spacelike regions. Second, the EE depends both on the number of species of matter fields, whose entanglement should reproduce the BH entropy, and on the value of the ultraviolet (UV) cut-off needed to regularize the divergences arising owing to the presence of a sharp boundary between the accessible and inaccessible regions of spacetime; conversely, the $\mathrm{BH}$ entropy should be universal.

A possible shortcut for circumventing these difficulties is to consider gravity theories with CFT duals and to make use of the AdS/CFT correspondence to identify the black hole EE with the EE of the dual CFT. This approach allows to reduce the computation of the black hole EE to calculations in a field theory where spacetime geometry is not dynamical.

In this paper we use this approach to compute the EE of two-dimensional (2D) and three-dimensional (3D) AdS black holes. Two- and three-dimensional AdS gravity allows for a dual description in terms of 2D CFT. The calculation of the black hole EE is reduced to the calculation of the EE of a 2D CFT, for which very simple expressions are known.

We derive explicit formulas for the entanglement entropy of 2D and 3D AdS black holes. In both cases, the leading term of the $\mathrm{EE}$ in the large black hole mass expansion reproduces exactly the $\mathrm{BH}$ entropy, whereas the subleading term behaves logarithmically.

This work is mainly based on [18,19] and represents an extended version of them. The structure of the paper is the following. In Section 2 we review some basic facts about EE in field theory, in particular CFT, and in black hole physics. In Section 3 we discuss the EE of 2D AdS black holes. In Section 4 we discuss the EE of 3D AdS black holes.

\section{Review of Entanglement Entropy}

\subsection{Entanglement in $Q F T$}

Quantum entanglement gives a measure of spatial correlations between parts of a system and it is measured by the entanglement entropy. In this paper we are mainly interested in the EE of a quantum field theory (QFT). In particular, we consider, following the discussion in [20], a field theory in 1+1 dimensions. Let $\phi \in[0, \Sigma[$ be the spacelike coordinate of a $2 \mathrm{D}$ universe. When only a spacelike 
slice is accessible for measurement, we lose information about the degrees of freedom localized in the complementary region. The subsystem where measurements are performed is $Q=[0, \gamma[$. The degrees of freedom in the complementary region $P=[\gamma, \Sigma[$ have to be traced over. EE turns out to be infinite, but it can be regularized by introducing an ultraviolet cutoff $\varepsilon$ necessary to regularize the divergence originated by the presence of a sharp boundary separating the region $P$ from the region $Q$.

The density matrix describing the subsystem $Q$, after tracing over the variables on $P$, is given by [20]

$$
\rho_{X X^{\prime}}=\int \mathcal{D} Y \Psi_{X Y} \Psi_{Y X^{\prime}}^{*}
$$

where the wave function of the system is

$$
\Psi_{X Y} \propto \int \mathcal{D} \Phi e^{-S(\Phi)}
$$

Here $\Phi$ denotes a complete collection of local fields on our theory and $X, Y$ are ordinary c-number functions. We take $\Phi=X$ on $Q$ and $\Phi=Y$ on $P$. The von Neumann entropy corresponding to the density matrix (1) is calculated using the replica trick

$$
S=-\operatorname{Tr} \rho \ln \rho=-\left.\frac{\partial}{\partial n} \operatorname{Tr} \rho^{n}\right|_{n=1} .
$$

We first evaluate $\operatorname{Tr} \rho^{n}$, differentiate it with respect to $n$ and finally take the limit $n \rightarrow 1$ ( $\rho$ is normalized such that $\operatorname{Tr} \rho=1$ ).

As discussed also in [21], $\operatorname{Tr} \rho^{n}$ can be computed in terms of path-integrals on an $n$-sheeted Riemann surface $\mathcal{R}_{n}$ :

$$
\operatorname{Tr} \rho^{n}=\frac{1}{Z_{1}^{n}} \int_{\mathcal{R}_{n}} \mathcal{D} \Phi e^{-S(\phi)} \equiv \frac{Z_{n}}{Z_{1}},
$$

where $Z_{n}$ is the partition function on a space obtained by gluing $n$ copies of the original space.

\subsection{Entanglement Entropy of a $2 D C F T$}

Using the general formulation described in Section 2.1, one can in principle compute the EE of any QFT. In general such a computation is rather involved, but it becomes much simpler in the case of a 2D conformal field theory. This is because the conformal symmetry can be used to determine the form of the correlation functions of the theory [20-22]. The resulting expression for the EE of the CFT depends on the topology of the $2 \mathrm{D}$ universe. For a $2 \mathrm{D}$ spacetime with the topology of the cylinder $\mathcal{C}$ shown in Figure 1a the EE turns out to be [20-22]:

$$
S_{\text {ent }}^{(\mathcal{C})}=\frac{c+\bar{c}}{6} \ln \left(\frac{\Sigma}{\varepsilon \pi} \sin \frac{\pi \gamma}{\Sigma}\right)
$$

where $c$ and $\bar{c}$ are the central charges of the 2D CFT and $\varepsilon$ is the UV cut-off. Thus, Equation (5) gives the EE for a $2 \mathrm{D}$ CFT on the cylinder $\mathcal{C}$, i.e. for a CFT at zero temperature and with a spacelike dimension with $S^{1}$ topology. 
For $\Sigma \gg \gamma$ the compact spacelike dimension becomes infinite, the spacetime has the topology of the plane shown in Figure $1 \mathrm{~b}$ and the EE is independent of $\Sigma$. In this limit Equation (5) gives the EE for a 2D CFT at zero temperature on the plane $\mathcal{P}$ [20-22]:

$$
S_{\text {ent }}^{(\mathcal{P})}=\frac{c+\bar{c}}{6} \ln \frac{\gamma}{\varepsilon}
$$

Figure 1. The three different forms of the $2 \mathrm{D}$ spacetime. $\phi$ is the spacelike coordinate and $t$ is the timelike one.

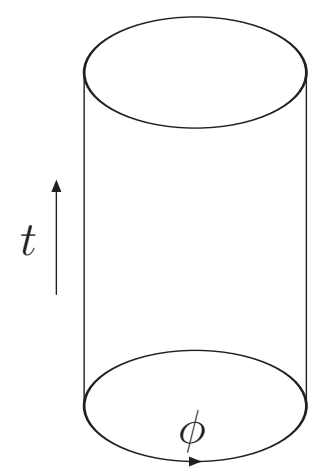

a) Cylinder $\mathcal{C}$

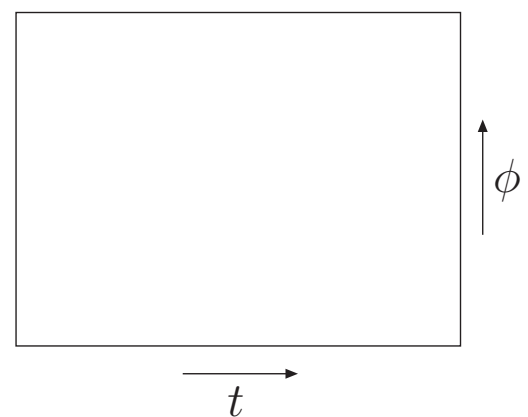

b) Plane $\mathcal{P}$

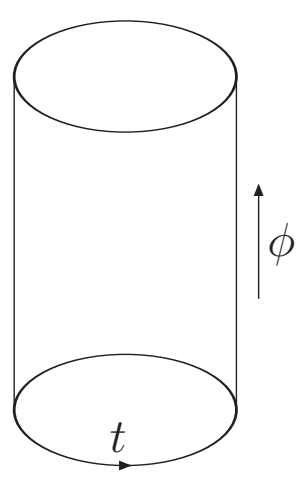

c) Cylinder $C$

We can also consider a 2D CFT at finite temperature $T=1 / \beta$ and a noncompact spacelike dimension. The spacetime has the topology of the cylinder $C$ represented in Figure 1c and the EE turns out to be [21]:

$$
S_{\text {ent }}^{(C)}=\frac{c+\bar{c}}{6} \ln \left(\frac{\beta}{\varepsilon \pi} \sinh \frac{\pi \gamma}{\beta}\right)
$$

One could also consider a spacetime with the topology of a torus $\mathcal{T}$. In this case the EE has not a universal form but depends on the details of the 2D CFT [23].

It is important to stress that the cylinder $C$ can be obtained as the limiting case of a torus $\mathcal{T}(\beta, \gamma)$ with cycles of length $\beta$, $\gamma$ when $\gamma \gg \beta$. In Section 4.6 we will use this feature to relate the thermal entropy of a CFT on the torus with the EE of a CFT on the cylinder $C$.

\subsection{AdS/CFT Correspondence and UV/IR Connection}

In Section 4 of this paper we will compute the EE of the BTZ black hole through an approach based on the holographic principle. In particular, we will use the AdS/CFT correspondence and the UV/IR relation, which are introduced in this section.

According to the holographic principle, suggested by 't Hooft [24] and Susskind [25], a bulk theory with gravity describing a macroscopic region is equivalent to a boundary theory without gravity living on the boundary of that region.

One of the most fruitful realizations of the holographic principle is the AdS/CFT correspondence, which was conjectured by Maldacena [26] in 1997. The $\mathrm{AdS}_{d+1} / \mathrm{CFT}_{d}$ correspondence states that each field $\Phi$ propagating in a $(d+1)$-dimensional anti-de Sitter spacetime is related, through a one to one 
correspondence, to an operator $\mathcal{O}$ in a $d$-dimensional conformal field theory defined on the boundary of that spacetime.

Essentially, the AdS/CFT correspondence can be interpreted as a relation between partition functions in the bulk and correlation functions on the boundary. The gravity partition function in the bulk turns out to be equal to the correlation functions of the operators $\mathcal{O}$ on the boundary:

$$
Z_{\text {bulk }}\left[\Phi_{0}(\vec{x})\right]=\left\langle e^{\int d^{4} x \Phi_{0}(\vec{x}) \mathcal{O}(\vec{x})}\right\rangle_{\text {boundary }}
$$

where the $d$-components of the variable $\vec{x}$ parametrize the boundary of $\operatorname{AdS}_{d+1}$ and $\Phi_{0}(\vec{x})$ is an arbitrary function specifying the boundary values of the field $\Phi(\vec{x}, z)$, with $z$ defined in the bulk. A similar relationship between fields in $\mathrm{AdS}_{d+1}$ and operators in $\mathrm{CFT}_{d}$ also exists for non-scalar fields, including fermions and tensors in anti-de Sitter spacetime.

The AdS/CFT correspondence implies that we can describe a boundary quantum field theory in terms of a bulk gravity theory. In particular infrared (IR) effects in the bulk theory describing a $(d+1)$-dimensional anti-de Sitter spacetime correspond to ultraviolet (UV) effects in the $d$-dimensional conformal field theory defined on the boundary: we call this relation the UV/IR connection [27,28].

Following Susskind and Witten in [27], we introduce an infrared regulator for the area of the boundary of the $\operatorname{AdS}_{d+1}$ spacetime, which is infinite. To do so, we replace the boundary (at $r=1$ in cavity coordinates or at $z=0$ in Poincaré coordinates) with a sphere at $r=1-\delta$ or, equivalently, at $z=\delta$, where $\delta$ is a small number acting as UV regulator of the CFT. The radius $R$ of the sphere turns out to be $R \sim \frac{\ell}{\delta}$ ( $l$ is the AdS length) therefore the area of the boundary diverges as $\delta \rightarrow 0$ and we can interpret $l / \delta$ as an IR regulator in the bulk theory.

This UV/IR connection is at the heart of the holographic requirement that the number of degrees of freedom should be of order the area of the boundary measured in Planck units [29].

\subsection{Entanglement Entropy of Black Holes}

As already mentioned in the Introduction, in the last years the notion of EE has been used with success as a tool for understanding quantum phases of matter, e.g., for spin chains and quantum liquids [1-4]. EE turned out to be very useful for investigating general features of quantum field theory and its relationship with gravity. In particular, the AdS/CFT correspondence has been used to calculate the EE of CFTs with gravitational duals, c-theorems have been shown to hold also for the EE and approximate formulas have been derived for the EE of generic QFTs in any dimension [5-7,30,31].

Because of its geometric nature, $\mathrm{EE}$ is also a natural candidate for trying to tackle one of the most difficult problems of black hole physics: the microscopic origin of the Bekenstein-Hawking entropy. The notion of quantum entanglement comes naturally in the play, since the horizon of a black hole divides spacetime into two subsystems, such that observers inside the horizon cannot communicate the results of their measurements to observers outside. This led 't Hooft to conjecture in [8] that the BH black hole entropy could be understood in terms of the quantum entanglement of matter in the black hole geometry [9-16]. Further support to this conjecture comes from the fact that both the Bekenstein-Hawking and the entanglement entropy scale as the area of the boundary. In [8] 't Hooft showed that the modes of a quantum field in the vicinity of a black hole horizon should be cut off due to gravitational effects. Although a detailed calculation of the physics associated with this phenomenon 
is quit difficult, it is possible to model such effects by imposing, within a distance $h$ from the horizon, a "brick wall" cut-off beyond which all modes of a quantum field must vanish. The model avoids the infinite piling up of modes near the horizon and the result for the EE does not depend upon either the mass or the charge of the source and is therefore a property only of the horizon. On the other hand, the EE depends both on the number $Z$ of species of matter fields localized in the black hole geometry and on the value $h$ of the UV cutoff, which has to be tuned to the value $h=\sqrt{Z / 90 \pi}$ if one wants to recover the Bekenstein-Hawking result. This is a problem, since the BH entropy should be universal, independent of both matter fields and UV cut-off.

Another basic, conceptual, problem is the difficulty to relate the usual statistical interpretation of the BH entropy - in terms of black hole microstates-with the meaning of EE, which measures the observer's lack of information about the quantum state of the system in inaccessible regions of spacetime. This problem becomes even more severe when one goes beyond the semiclassical approximation. As mentioned in the Introduction, the very notion of EE for pure quantum gravity is not easy to define: the main obstruction comes from the fact that in the Euclidean quantum gravity formulation [17] only the boundary value of the metric can be fixed a priori, whereas the usual flat-space notion of EE requires to fix lengths in bulk spacelike regions. Some of this conceptual problems may look somehow milder using Sakharov's induced gravity approach $[32,33]$, but the fundamental issue is still there.

A possible way out of these difficulties is to consider gravity theories with CFT duals (see e.g., [34]) and to make use of the AdS/CFT correspondence to identify the black hole EE with the EE of the dual CFT.

There are several advantages in pursuing this approach. As explained in Section 2.2, at least for CFTs in two dimensions, explicit and simple formulas for the EE are known. Moreover, one can define the $\mathrm{EE}$ of a gravity configuration in terms of the EE of a field theory in which spacetime geometry is not dynamic, thus avoiding the usual difficulties of the Euclidean quantum gravity approach. Last but not least, the use of the AdS/CFT correspondence allows to solve the universality problem: one can identify in a natural way the UV cut-off $h$ with the the AdS length $l$ and the number of matter fields $Z$ with the central charge $c$ of the CFT.

There are two main drawbacks of this approach. The first is related to the fact that the AdS/CFT correspondence is holographic. Spatial correlations in the bulk gravity theory are codified in a highly nonlocal way in the correlations of the boundary CFT. This is particularly evident in the UV/IR relation, which relates large distances on AdS space with the short distances behavior of the boundary CFT $[27,28]$. Because of this difficulty, the AdS/CFT correspondence has been only partially fruitful for understanding the EE of gravitational configurations, in particular black holes. Some progress in this direction has been achieved in the general case in [11,12,30]. Strangely enough, the AdS/CFT correspondence has been used with much more success in the reversed way, i.e., to compute the EE of boundary CFTs in terms of bulk geometric quantities [5-7,35-37].

The second strong limitation of the holographic approach is that it works only for gravity models with CFT duals. The most important class of such models is AdS gravity. In Section 3 of this paper we will study the EE of 2D AdS black holes, while in Section 4 we will consider the 3D Bañados-Teitelboim-Zanelli (BTZ) black hole. 


\section{2D AdS Black Holes}

Two-dimensional models of gravity have been used as toy models for dealing in a simplified context with complicated problems of black hole physics and quantum gravity. In particular, they can be used to describe the radial modes (the $S$-wave sector) of four- and higher-dimensional static black holes. In this section we derive, using the AdS/CFT correspondence, a simple form for the EE of 2D AdS black holes. This will turn out to be possible because of peculiarity of 2D AdS gravity, namely the fact that 2D gravity can be considered as induced by quantum fluctuations of matter fields.

\subsection{Entanglement Entropy of the 2D Black Hole}

2D AdS black holes are classical solutions of a 2D dilaton-gravity theory, which in the simplest case is described by the action $A=\int d^{2} x \sqrt{g} \Phi\left(R+2 / \ell^{2}\right)$, where the AdS length $\ell$ is related to the cosmological constant $\lambda$ of the AdS spacetime $\left(\lambda=1 / \ell^{2}\right)$ and $\Phi$ is a scalar called dilaton. The $2 \mathrm{D}$ AdS black hole solutions can be written as follows [38]:

$$
d s^{2}=-\frac{1}{\ell^{2}}\left(r^{2}-r_{h}^{2}\right) d t^{2}+\ell^{2}\left(r^{2}-r_{h}^{2}\right)^{-1} d r^{2}, \quad \Phi=\frac{r}{G_{2} \ell},
$$

where $G_{2}$ is the dimensionless $2 \mathrm{D}$ Newton constant and $r_{h}$ is the horizon radius.

The black hole mass, temperature and Bekenstein-Hawking (BH) entropy are given by [38]

$$
M=\frac{r_{h}^{2}}{2 G_{2} \ell^{3}}, \quad T=\frac{r_{h}}{2 \pi \ell^{2}}, \quad S_{B H}=\frac{2 \pi r_{h}}{G_{2} \ell} .
$$

The 2D gravity theory has a dual description in terms of a chiral CFT with with central charge [39-42]

$$
c=\frac{12}{G_{2}} .
$$

This AdS/CFT correspondence has been used to give a microscopical meaning to the thermodynamic entropy of the 2D AdS black hole. The BH entropy (10) can be also reproduced by counting states in the dual CFT [39-42].

It has been observed that in two dimensions black hole entropy can be ascribed to quantum entanglement if 2D Newton constant is wholly induced by quantum fluctuations of matter fields $[10,32,33,43]$. But Equation (11) tells us that the 2D Newton constant is induced by quantum fluctuations of the dual CFT. It follows that in the semiclassical approximation the black hole EE should be identified with the EE of the vacuum for the 2D CFT with central charge given by Equation (11) in the black hole geometry (9). The black hole exterior and interior should be identified respectively with an observable region $Q$ of length $\gamma$ and an unobservable region $P$ of length $\Sigma-\gamma$ in the 2D universe described above, where the CFT degrees of freedom live.

However, two obstacles prevent a direct application of Equation (5):

(1) Equation (5) holds for a 2D flat spacetime, whereas we are dealing with a curved 2D background.

(2) The calculations leading to Equation (5) are performed for a spacelike slice $Q$, whereas in the black hole case there is no global notion of a spacelike coordinate, owing to the coordinate singularities at $r=r_{h}$ (the location of the horizon) and at $r=\infty$ (the location of the timelike asymptotic boundary of the AdS spacetime). 
Owing to these geometric features, in the black hole case we cannot give a direct meaning to both the measures $\gamma$ and $\Sigma-\gamma$ of the subsystems $Q$ and $P$.

The second difficulty can be circumvented using an appropriate coordinate system and a regularization procedure; the first difficulty can be solved using, instead of Equation (5), the formula derived by Fiola et al. in [10], which gives the EE of the CFT vacuum in the case of a curved background,

$$
S_{\text {ent }}=-\frac{c}{6}\left(\left.\varrho\right|_{\text {boundary }}-\ln \frac{\varepsilon}{\Sigma}\right)
$$

where $c$ is the central charge (11) and $e^{2 \varrho}$ is the conformal factor of the metric in the coordinate system used to define the CFT vacuum. Also notice that we are using the formula of [10] with reversed sign. The AdS black hole has to be considered as the vacuum seen by the observer using the black hole coordinates. This observer sees the CFT vacuum as filled with thermal radiation with negative flux [38]. In this coordinate system the black hole metric (9) takes the form [38]

$$
d s^{2}=\frac{r_{h}^{2}}{\ell^{2}} \frac{\left(-d t^{2}+d \sigma^{2}\right)}{\sinh ^{2}\left(\frac{r_{h} \sigma}{\ell^{2}}\right)} .
$$

Notice that in Equation (12) we have contributions from only one sector (e.g., right movers) of the CFT. The CFT dual to 2D dilaton gravity is the one chiral sector of a 2D CFT, as shown in $[41,42]$. The 2D AdS black hole is dual to an open string with appropriate boundary conditions. These boundary conditions are such that only one sector of the 2D CFT is present.

The coordinate system $(t, \sigma)$ covers only the black hole exterior. Working in Euclidean space the 2D manifold has only a boundary at $\sigma=0$, corresponding to $r=\infty$, i.e. to the timelike conformal boundary of the 2D AdS space. The conformal factor of the metric (13), as well as the entanglement entropy (12), blows up on the $\sigma=0$ boundary of the AdS spacetime. The simplest regularization procedure is to consider a regularized boundary at $\sigma=\varepsilon$. Here $\varepsilon$ plays the role of a UV cut-off for the coordinate $\sigma$, which is the natural spacelike coordinate of the dual CFT. But $\varepsilon$ is also an IR cut-off for the coordinate $r$, which is the natural spacelike coordinate for the 2D AdS black hole.

The regularized boundary is at finite proper distance from the horizon. Since $\varepsilon$ also acts as an IR regulator, the presence of the IR cut-off $\Sigma$ in Equation (12) turns out to be redundant. The EE still depends on the UV cut-off $\varepsilon$. The AdS/CFT correspondence enables us to identify $\varepsilon$ as the UV cut-off of the CFT: $\varepsilon \propto \ell$. The proportionality factor can be determined by requiring that the analytical continuation of Equation (14) below is invariant under the transformation $\gamma \rightarrow \Sigma-\gamma$ which exchanges the inside and outside regions. This requirement fixes $\varepsilon=\pi \ell$.

Putting all together we obtain from Equation (12) the EE of the 2D CFT in the classical black hole geometry:

$$
S_{\text {ent }}=\frac{c}{6} \ln \left(\frac{\ell}{\pi r_{h}} \sinh \frac{\pi r_{h}}{\ell}\right)
$$

which has to be identified with the EE of the 2D AdS black hole. The entanglement entropy (14) has the expected behavior as a function of the horizon radius $r_{h}$ or, equivalently, of the black hole mass $M$. $S_{\text {ent }}$ becomes zero in the AdS ground state, for $r_{h}=0(M=0)$, whereas it grows monotonically for $r_{h}>0$ $(M>0)$. 
The black hole EE (14) takes a form very similar to the EE of a 2D CFT at finite temperature (see Equation (7)). On the other hand, our formula (14) differs from the EE for a CFT at zero temperature (see Equation (5)) by the exchange of hyperbolic with trigonometric functions. This indicates that Equation (5) can be obtained as the analytic continuation $r_{h} \rightarrow i r_{h}$ of our formula (14), i.e. by considering the solution (9) with negative mass. The analytically continued solution is given by Equation (9) with $r_{h}^{2}<0$. In the conformal gauge the solution reads now $d s^{2}=\left[r_{h} /\left(\ell \sin \left(r_{h} \sigma / \ell^{2}\right)\right]^{2}\left(-d t^{2}+d \sigma^{2}\right)\right.$.

In terms of the 2D CFT we have to trace over the degrees of freedom outside the spacelike slice $\varepsilon<\sigma<\pi \ell^{2} / 2 r_{h}$. Applying Equation (12) to the case of a spacelike slice with two boundary points and redefining appropriately the UV cut-off $\varepsilon$, we get

$$
S_{\text {ent }}=\frac{c}{6} \ln \left(\frac{\Sigma}{\pi \varepsilon} \sin \frac{\pi r_{h}}{\Sigma}\right) \text {. }
$$

Thus, the EE of the 2D CFT in the curved background given by the AdS solution with negative mass has exactly the form given by Equation (5), with the horizon radius $r_{h}$ playing the role of $\gamma$. The requirement that Equation (15) is the analytic continuation of Equation (14) fixes, as previously anticipated, the proportionality factor between $\varepsilon$ and $\ell$ in the calculation leading to Equation (14).

\subsection{Large Black Hole Mass Behavior}

Let us now consider the large mass behavior $r_{h} \gg \ell$ (macroscopic black holes) of Equation (14):

$$
S_{\text {ent }}=\frac{2 \pi r_{h}}{G_{2} \ell}-\frac{2}{G_{2}} \ln \frac{r_{h}}{\ell}+\mathcal{O}(1)=S_{B H}-\frac{2}{G_{2}} \ln S_{B H}+\mathcal{O}(1)
$$

We have obtained the remarkable result that the leading term in the large mass expansion of the black hole EE reproduces exactly the BH entropy. In this regime, with thermal length given by $\beta=1 / T \ll \ell$, thermal correlations dominate and the EE becomes Gibbs entropy. The subleading term has the universally predicted $\ln S_{B H}$ behavior for the quantum corrections to the $\mathrm{BH}$ result. Notice that, although it is universally accepted that the quantum corrections to the black hole entropy behave as $\ln S_{B H}$ [44-52], there is no general agreement about the value of the prefactor of this term. Equation (16) fixes the prefactor of $\ln S_{B H}$ in terms of the 2D Newton constant. Our result, which contradicts some previous results supporting a $G_{2}$-independent value of the prefactor, is consistent with our approach, which considers 2D gravity as induced from the quantum fluctuations of a CFT with central charge $c=12 / G_{2}$. The subleading term in Equation (16) has also the universal behavior shared with other systems described by 2D QFTs, such as one-dimensional statistical models near the critical point (with the black hole radius $r_{h}$ corresponding to the correlation length) or free scalar fields [21,31].

For $\beta \gg \ell$ quantum correlations dominate, but we expect our semiclassical description, which also neglects back-reaction, to break down at $\beta \sim \ell$. In this regime it is likely that a phase transition, analogue to the Hawking-Page transition $[53,54]$ in $4 \mathrm{D}$ gravity, takes place.

\section{BTZ Black Holes}

There are several arguments indicating that our derivation of the EE of 2D AdS black holes could be extended to black holes in 3D AdS spacetime, i.e. to the BTZ black hole. Pure gravity in three spacetime 
dimensions has the same degrees of freedom (DOF) of 2D dilaton gravity, that is only global DOFs and no propagating gravitons. Moreover, 2D AdS dilaton gravity can be considered as a circular symmetric dimensional reduction of 3D AdS gravity with the dilaton parametrizing the radius of the transverse circle. The 2D AdS black hole discussed in Section 3 of this paper can be obtained as dimensional reduction of the BTZ black hole with vanishing angular momentum $J$. The thermodynamic parameters (mass, temperature and entropy) characterizing the $J=0$ BTZ black hole match exactly those pertinent to the 2D AdS black hole, given by Equation (10), once one expresses the 3D Newton constant $G_{3}$ in terms of the 2D Newton constant $G_{2}$ and of the de Sitter length $\ell$.

In the case of 2D AdS gravity, the dual theory has the form of a chiral CFT, whereas in the 3D case, owing to different boundary conditions, the dual theory is a 2D CFT with both left and right movers.

In this section of the paper we investigate quantum entanglement in the context of 3D AdS gravity, in particular the Bañados-Teitelboim-Zanelli (BTZ) black hole, using the $\mathrm{AdS}_{3} / \mathrm{CFT}_{2}$ correspondence. We will tackle the problem using a standard method for studying correlations in QFT: we introduce in the boundary 2D CFT two external length-scales, a thermal wavelength $\beta=1 / T$ (with $T$ temperature of the CFT) and a spatial length $\gamma$, which is the measure of the observable spatial region of our 2D universe. Varying $\beta$ we can probe thermal correlations of the CFT at different energy scales, whereas varying $\gamma$ we can probe the spatial correlations at different length scales. The EE of a QFT gives information about the spatial correlations of the theory. It follows that the EE of a 2D CFT, which is the holographic dual of 3D gravity, should contain information about bulk quantum gravity correlations.

We will show that the AdS/CFT correspondence, and in particular the UV/IR relation, allows us to identify in a natural way $\beta$ and $\gamma$ in terms of the two fundamental bulk length-scales, the horizon of the BTZ black hole $r_{+}$and the AdS length $\ell$. This enables us, using the expression (5), (6), (7) for the EE of 2D CFTs and modular symmetry, to associate a "holographic" EE to regularized 3D AdS, to the BTZ black hole and to 3D AdS with conical singularities.

\section{1. $A d S_{3}$ Gravity and Dual $\mathrm{CFT}_{2}$}

Classical, pure $\mathrm{AdS}_{3}$ gravity is described by the action

$$
A=\frac{1}{16 \pi G_{3}} \int d^{3} x\left(R+\frac{2}{\ell^{2}}\right),
$$

where $\ell$ is the de Sitter length and $G_{3}$ is the 3D Newton constant. The exact form of the 2D CFT dual to 3D AdS gravity still remains a controversial point [55-57]. However, in the large $N$ regime (central charge $c \gg 1$ ), i.e. in the region of validity of the gravity description, we know that the dual CFT has central charges [58]

$$
c=\bar{c}=\frac{3 \ell}{2 G_{3}} .
$$

$\mathrm{AdS}_{3}$ classical gravity allows for three kinds of spacetime configurations. These are solutions of the action (17) and can be classified in terms of orbits (elliptic, hyperbolic, parabolic) of the $\mathrm{SL}_{2}(\mathbb{R})$ group manifold $[55,59,60]$. The solutions corresponding to elliptic orbits can be written as

$$
d s^{2}=-\frac{1}{\ell^{2}}\left(r^{2}+r_{+}^{2}\right) d t^{2}+\left(r^{2}+r_{+}^{2}\right)^{-1} \ell^{2} d r^{2}+\frac{r^{2}}{\ell^{2}} d \phi,
$$


where $0 \leq t \leq \beta, 0 \leq \phi \leq 2 \pi \ell, 0 \leq r<\infty$ and $r_{+}$is a constant. The corresponding 3D Euclidean space has a contractible cycle in the spatial $\phi$-direction. For generic values of $r_{+}$we have therefore a conical singularity in this direction. Only for $r_{+}=\ell$ the conical singularity disappears and the manifold becomes a nonsingular 3D AdS space at finite temperature $1 / \beta$. The conformal boundary of the 3D spacetime is a torus with cycles of length $\beta$ and $2 \pi \ell$. Correspondingly, the dual CFT will live in the torus $\mathcal{T}(\beta, 2 \pi \ell)$. The $\mathrm{CFT}$ on the cylinder $\mathcal{C}$ discussed in Section 2.2 can be obtained in the limit $\beta \gg \ell$. This corresponds to consider $-\infty<t<\infty$ and $0 \leq \phi \leq 2 \pi \ell$.

The classical solutions of $3 \mathrm{D}$ gravity corresponding to hyperbolic orbits of $\mathrm{SL}_{2}(\mathbb{R})$ are

$$
d s^{2}=-\frac{1}{\ell^{2}}\left(r^{2}-r_{+}^{2}\right) d t^{2}+\left(r^{2}-r_{+}^{2}\right)^{-1} \ell^{2} d r^{2}+\frac{r^{2}}{\ell^{2}} d \phi^{2} .
$$

Now the 3D Euclidean manifold has a contractible cycle in the $t$-direction. For generic values of $\beta$ and $r_{+}$we have therefore a conical singularity in this direction. Only for $\beta=\beta_{H}$, where $\beta_{H}$ is the inverse Hawking temperature

$$
\beta_{H}=\frac{1}{T_{H}}=\frac{2 \pi \ell^{2}}{r_{+}}
$$

the conical singularity can be removed and the space describes the Euclidean BTZ black hole. The black hole has horizon radius $r_{+}$, and mass and Bekenstein-Hawking entropy given by

$$
M=\frac{r_{+}^{2}}{8 G_{3} \ell^{2}}, \quad S_{B H}=\frac{\mathcal{A}}{4 G_{3}}=\frac{\pi r_{+}}{2 G_{3}} .
$$

Also in this case the conformal boundary of the 3D spacetime is the torus with cycles of length $\beta_{H}$ and $2 \pi \ell$, and the dual CFT will live on $\mathcal{T}\left(\beta_{H}, 2 \pi \ell\right)$. The CFT on the cylinder $C$ discussed in Section 2.2 can be obtained in the limit $\ell \gg \beta_{H}$. This corresponds to consider a CFT at finite temperature, $0 \leq t \leq \beta_{H}$, with noncompact spacelike dimension $-\infty<\phi<\infty$. In terms of the $3 \mathrm{D}$ bulk theory this corresponds to a macroscopic black hole with $r_{+} \gg \ell$.

The separating element between the two classes of solutions discussed above corresponds to parabolic orbits of $\mathrm{SL}_{2}(\mathbb{R})$,

$$
d s^{2}=-\frac{1}{\ell^{2}} r^{2} d t^{2}+\frac{\ell^{2}}{r^{2}} d r^{2}+\frac{r^{2}}{\ell^{2}} d \phi^{2}, \quad-\infty<t<\infty .
$$

The solution can be seen as the $r_{+}=0$ ground state of the BTZ black hole, i.e. the $M=0, T_{H}=0$ solution.

For $r_{+} \neq \ell$ the solution (19) has a conical singularity not shielded by an event horizon [59,60]. The conical singularity can also be thought of as originated by a pointlike source of mass $m$. In the spectrum of $\mathrm{AdS}_{3}$ gravity these solutions are located between the NS vacuum, $r_{+}=\ell$, and the RR vacuum, $r_{+}=0$. Therefore we will consistently take $0 \leq r_{+} \leq \ell$.

If we rescale the coordinates in Equation (19),

$$
r \rightarrow \frac{r_{+}}{\ell} r, \quad t \rightarrow \frac{\ell}{r_{+}} t, \quad \phi \rightarrow \frac{\ell}{r_{+}} \phi
$$

the metric becomes

$$
d s^{2}=-\left(\frac{r^{2}}{\ell^{2}}+1\right) d t^{2}+\left(\frac{r^{2}}{\ell^{2}}+1\right)^{-1} d r^{2}+\frac{r^{2}}{\ell^{2}} d \phi
$$


The previous expression describes thermal $\mathrm{AdS}_{3}$ in global coordinates but, owing to the rescaling of the coordinates, we have now $0 \leq \phi \leq 2 \pi \Gamma \ell$, with $\Gamma=r_{+} / \ell$. The spacetime has a conical singularity originated by a deficit angle $2 \pi(1-\Gamma)=2 \pi\left(1-r_{+} / \ell\right)=2 \pi\left(1-2 \pi \ell / \beta_{c o n}\right)$, where we have introduced

$$
\beta_{\text {con }}=\frac{2 \pi \ell^{2}}{r_{+}} \text {. }
$$

$\beta_{\text {con }}$ is the analogous of the inverse Hawking temperature $\beta_{H}$ and characterizes the conical singularity. In the case of solution (20), setting $\beta=\beta_{H}$ eliminates the conical singularity, whereas for solution (19) we get a regular manifold ( $\mathrm{AdS}_{3}$ at finite temperature) for $\beta_{\text {con }}=2 \pi \ell$.

In order to find the holographic EE of the solution (19), (20) and (23), we have to discuss first the modular symmetry of the 2D CFT dual to 3D AdS gravity.

\subsection{Modular Invariance}

It is well known that the partition function of a 2D CFT on the complex has to be invariant for transformation of the modular group $\mathrm{PSL}_{2}(\mathbb{Z})$

$$
\tau \rightarrow \frac{a \tau+b}{c \tau+d}
$$

where $a, b, c, d$ are integers satisfying $a d-b c=1, \tau=\omega_{2} / \omega_{1}$ is the modular parameter of the torus and $\omega_{1,2}$ are the periods of the torus. For simplicity we will take $\omega_{1}=\gamma$ real and $\omega_{2}=i \beta$ purely imaginary. We are mainly interested in the modular transformation of the torus

$$
\tau \rightarrow-\frac{1}{\tau}
$$

3D spaces which are asymptotically AdS are locally equivalent. The asymptotic form of the coordinate transformations mapping the various spaces can be used to map one into the other the tori describing the associated conformal boundaries. For our discussion the relevant elements are the Euclidean BTZ black hole at Hawking temperature $1 / \beta_{H}, \mathrm{AdS}_{3}$ space with deficit angle $2 \pi\left(1-2 \pi \ell / \beta_{\text {con }}\right)$ and $\mathrm{AdS}_{3}$ at finite temperature $1 / \beta_{H}$. It will turn out that the boundary tori associated with these three spaces are related by modular transformations of the torus.

Let us briefly review the well-known duality between the BTZ black hole and $\mathrm{AdS}_{3}$ at finite temperature [34,61]. To this purpose, we use the fact that the Euclidean BTZ solution (20) with periodicity $t \sim t+\beta_{H}, \phi \sim \phi+2 \pi \ell$ can be mapped by a diffeomorphism into $\mathrm{AdS}_{3}$ in Poincaré coordinates

$$
d s^{2}=\frac{1}{x^{2}}\left(d y^{2}+d z d \bar{z}\right)
$$

where $z$ is a complex coordinate.

In the asymptotic $r \rightarrow \infty(x \rightarrow 0)$ region the map between the BTZ black hole and $\mathrm{AdS}_{3}$ in Poincaré coordinates is

$$
z=\exp \left[\frac{2 \pi}{\beta_{H}}(\phi+i t)\right]
$$

In order to have a natural periodicity, we introduce a new complex variable $w$

$$
z=\exp (-2 \pi i w)
$$


so that $w=(-t+i \phi) / \beta_{H}$. One can now easily realize that the asymptotic conformal boundary of the BTZ black hole is a complex torus with metric $d s^{2}=d w d \bar{w}$. The periodicity of the imaginary $\left(\omega_{2}\right)$ and real $\left(\omega_{1}\right)$ part of $w$ are determined by the periodicity of $t, \phi: \omega_{2}=2 \pi i \ell / \beta_{H}, \quad \omega_{1}=1$. The modular parameter $\tau_{B T Z}=\omega_{2} / \omega_{1}$ of the torus is therefore

$$
\tau_{B T Z}=\frac{2 \pi i \ell}{\beta_{H}}
$$

Consider now Euclidean $\mathrm{AdS}_{3}$ at finite temperature, described by the metric (25) with periodicity $t \sim t+\beta_{H}$ and $\phi \sim \phi+2 \pi \ell$. The $r \rightarrow \infty$ asymptotic form of the map between $\mathrm{AdS}_{3}$ at finite temperature and $\mathrm{AdS}_{3}$ in Poincaré coordinates is

$$
z=\exp \left(\frac{t-i \phi}{\ell}\right),
$$

whereas the coordinate $w$ in Equation (31) is now $w=\frac{1}{2 \pi \ell}(\phi+i t)$. The complex coordinate $w$ has now periodicity $\omega_{1}=1, \omega_{2}=i \beta_{H} / 2 \pi \ell$. The boundary of thermal $\mathrm{AdS}_{3}$ is a torus with modular parameter

$$
\tau_{A d S}=\frac{i \beta_{H}}{2 \pi \ell} .
$$

Hence the boundary torus of the BTZ black hole and that of thermal $\mathrm{AdS}_{3}$ are related by the modular transformation

$$
\tau_{A d S}=-\frac{1}{\tau_{B T Z}}
$$

Passing to consider the Euclidean solution with the conical singularity (19), we note that it is related to $\mathrm{AdS}_{3}$ just by the rescaling (24). This changes the periodicity of the coordinates, which becomes $t \sim t+2 \pi \ell, \phi \sim \phi+4 \pi^{2} \ell^{2} / \beta_{\text {con }}$. Because the coordinate transformation mapping the boundary torus of the conical singularity space into the boundary torus of $\mathrm{AdS}_{3}$ has the same form given by Equation (33), it follows that the periodicity of the coordinate $w$ is now $\omega_{1}=2 \pi \ell / \beta_{\text {con }}, \omega_{2}=i$. If we set $\beta_{\text {con }}=\beta_{H}$, the periodicities of the two tori are related by

$$
\omega_{2}^{c o n}=\frac{i}{\omega_{1}^{A d S}}, \quad \omega_{1}^{c o n}=\frac{i}{\omega_{2}^{A d S}} .
$$

The boundary torus of Euclidean $\mathrm{AdS}_{3}$ with conical singularity characterized by the deficit angle $2 \pi\left(1-2 \pi \ell / \beta_{H}\right)$ has the same modular parameter as that of $\mathrm{AdS}_{3}$ at temperature $1 / \beta_{H}$. Notice that, although the two manifolds have the same topology and the same boundary torus, they describe different three-geometries. The first is a singular one, whereas the latter is a perfectly well-behaved geometry. For this reason, one usually does not include $\mathrm{AdS}_{3}$ with conical singularities in the physical spectrum of the theory.

Because $\tau_{c o n}=\tau_{A d S}$, from Equation (35) it follows immediately that the boundary tori of $\mathrm{AdS}_{3}$ with conical defect $2 \pi\left(1-2 \pi \ell / \beta_{H}\right)$ and that of the BTZ black hole at inverse temperature $\beta_{H}$ are related by the modular transformation

$$
\tau_{c o n}=-\frac{1}{\tau_{B T Z}}
$$




\subsection{EE and the UV/IR Relation}

As a consequence of the AdS/CFT correspondence, the EE expressed in Equations (5), (6) and (7) should give information about bulk correlators. More precisely, one would expect the EE in Equation (5) to describe quantum correlations in the presence of conical singularity (19) and the EE in Equation (7) of the thermal CFT to describe the interplay between thermal and quantum correlations in the black hole background (20). The main obstacle to make the above relation precise is due to the holographic nature of the AdS/CFT correspondence. Spatial correlations in the bulk gravity theory are codified in the boundary CFT in a highly nonlocal way. Whereas the inverse temperature $\beta$ appearing in Equation (7) can be naturally identified as the inverse of the black hole temperature (21), the same is not true for the parameters $\gamma$ and $\varepsilon$ in Equations (5), (6) and (7).

Owing to the holographic nature of the correspondence, the bulk interpretation of these parameters requires careful investigation. As discussed in Section 2.3, the $\mathrm{AdS}_{d+1} / \mathrm{CFT}_{d}$ correspondence indicates a way to relate length scales on the boundary with length scales on the bulk, this is the UV/IR connection [27,28]. Infrared effects in bulk $\mathrm{AdS}_{d+1}$ gravity correspond to ultraviolet effects in the boundary $\mathrm{CFT}_{d}$, and vice versa.

The UV/IR connection allows to identify the UV cut-off $\varepsilon$ in Equation (6) as an IR regulator of $\operatorname{AdS}_{3}$ gravity $[27,28]$. This can be done in the usual way by using the dilatation isometry of the metric (23): $r \rightarrow \lambda r, t \rightarrow \lambda^{-1} t, \phi \rightarrow \lambda^{-1} \phi$. Equivalently, one can introduce "cavity coordinates" on $\mathrm{AdS}_{3}$ and show that $\varepsilon$ acts as an infrared regulator of the "area" of the $S^{1}$ boundary sphere [27]. In fact, the regularized radius of the $S^{1}$ is $R=\ell^{2} / \varepsilon$. The same is true in terms of the coordinate $r$ parametrizing $\operatorname{AdS}_{3}$ in the modified Poincaré form (23): cutting off the 2D CFT at length scale less than $\varepsilon$ implies an infrared cut-off on the radial coordinate of $\mathrm{AdS}_{3}, r<\Sigma$, where

$$
\Sigma=\frac{4 \pi^{2} \ell^{2}}{\varepsilon} \text {. }
$$

The length-scales $\gamma$ and $\varepsilon$ are defined up to a dimensionless multiplicative constant of $\mathcal{O}(1)$. In the following we will set this multiplicative constant equal to $2 \pi$.

The bulk interpretation of the parameter $\gamma$ in Equation (6) is not as straightforward as that of $\varepsilon$. $\gamma$ is not a simple external length scale we are using to cut off excitations of energy less than $1 / \gamma$. It is the length of a localized spacelike slice of the 2D space on which the CFT lives. On the other hand, owing to the holographic, nonlocal nature of the bulk/boundary correspondence, we expect that any localization of DOFs on the boundary will be lost by the correspondence with DOFs in the bulk. If any localization property of the observable slice $Q$ is lost in the boundary/bulk duality, $\gamma$ can only play the role of an upper bound above which spatial correlations for the boundary CFT are traced out. Because of the UV/IR connection, in $\mathrm{AdS}_{3}$ this will correspond to tracing out the bulk DOFs at small values of the radial coordinate $r$, i.e. for $r<\omega$, where

$$
\omega=\frac{4 \pi^{2} \ell^{2}}{\gamma} .
$$

It is important to stress that the bulk parameter $\omega$ has not the same physical meaning of the boundary parameter $\gamma$. Whereas $\gamma$ is the length of a spacelike slice, which is sharply separated from the observable region (hence it needs a UV regulator), $\omega$ has the much weaker meaning of a length scale below 
which spatial correlations are traced out. In particular in the $\mathrm{AdS}_{3}$ bulk there is no sharp boundary separating observable and unobservable regions. The bulk interpretation of $\gamma$, given Equation (39) is highly nontrivial and has the status of a conjecture, which is supported by the UV/IR relation. On the other hand, once one accepts the validity of Equation (39) from the UV/IR relation it follows immediately that tracing in the boundary for distances greater than $\gamma$ corresponds to tracing in the bulk for $r<\omega$. We can use this meaning of $\gamma$ and $\omega$ to interpret the EE (5), (6) and (7) as holographic entanglement entropies of gravitational configurations.

The AdS/CFT correspondence and the IR/UV connection allow us to give to the EE (6) a simple bulk interpretation: it is the EE of the regularized $\mathrm{AdS}_{3}$ spacetime (23), i.e. it gives a measure of the von Neumann entropy that arises when an IR cut-off $\Sigma$ is introduced and correlations are traced over for $r<\omega$. Substituting Eqs. (38) and (39) into Equation (6), we find $S_{\text {ent }}^{A d S}=\frac{c}{3} \ln \frac{\Sigma}{\omega}$ (we have used $\bar{c}=c$ ). The natural length scale for cutting off quantum bulk correlations is given by the AdS length $\ell: \omega=2 \pi \ell$. This means that we are considering curvature effects much smaller than $1 / \ell^{2}$. Using Equation (39), this allows the identification of the boundary parameter in terms of the AdS length $\ell$

$$
\gamma=2 \pi \ell
$$

The holographic EE of the regularized $\mathrm{AdS}_{3}$ spacetime

$$
S_{\text {ent }}^{A d S}=\frac{c}{3} \ln \frac{\Sigma}{\ell}
$$

has a simple geometric interpretation. Apart from a proportionality factor, it is the (regularized) proper length of the spacelike curve $t=$ const, $\phi=$ const. This can be easily shown integrating Equation (23) for $\ell \leq r \leq \Sigma$.

It is interesting to notice that the identification $\gamma=2 \pi \ell$ can be obtained without using the UV/IR connection, just assuming that in the large $N$ limit the mass/temperature relationship for the BTZ black hole exactly reproduces that of a thermal 2D CFT.

From Equations (21) and (22) one easily finds the mass/temperature relationship for the BTZ black hole,

$$
M=\frac{\pi^{2} \ell^{2}}{2 G_{3}} T_{H}^{2} .
$$

On the other hand, in the large temperature limit $\gamma \gg \beta$ the EE (7) reduces to the classical, extensive thermal entropy for an isolated system of length $\gamma$. The energy/temperature relationship for such a 2D CFT is given by

$$
E-E_{0}=\frac{c}{12} \pi \gamma\left(T_{+}^{2}+T_{-}^{2}\right)
$$

where $E_{0}$ is the energy of the vacuum and $T_{+}, T_{-}$are the temperatures for the right and left oscillators. Identifying the black hole mass $M$ with $E-E_{0}$ and the temperature $T_{H}=T_{+}=T_{-}$of the CFT thermal state with the Hawking temperature of the black hole, we easily find, comparing Equation (43) with Equation (42) and using Equation (18), $\gamma=2 \pi \ell$.

\subsection{Holographic EE of Conical Singularities}

Let us now consider the classical solution of 3D AdS gravity given by Equation (19), which describes 3D AdS spacetime with conical singularities. As explained in Section 4.2, solution (19) can be locally 
obtained applying a diffeomorphism to the AdS spacetime (23). This transformation is the "spacelike" counterpart of the "thermalization" mapping the metric (23) into the BTZ black hole. On the 2D conformal boundary of the 3D AdS spacetime this transformation is described by the map

$$
z=\exp \left(\frac{t-i \phi}{\beta}\right)
$$

$\beta$ is easily determined by first applying the transformation (33) mapping full $\operatorname{AdS}_{3}$ into (23) and then using the rescaling (24): $\beta=\beta_{\text {con }}$, where $\beta_{\text {con }}$ is given by Equation (26). In the limit $\beta_{\text {con }} \gg 2 \pi \ell$ (i.e. $\ell \gg r_{+}$) the map (44) corresponds to a plane/cylinder transformation that maps the CFT on the plane $\mathcal{P}$ into the CFT on the cylinder $\mathcal{C}$. Thus, this conformal transformation maps the EE of a CFT on the plane $\mathcal{P}$ into the EE of a CFT in the cylinder $\mathcal{C}$ [21], i.e. the EE of a CFT at zero temperature and noncompact spacelike dimension given by Equation (6) into the EE of a CFT at zero temperature with a compact spacelike dimension given by Equation (5). Correspondingly, the holographic EE of the regularized AdS spacetime becomes the holographic EE associated to $\mathrm{AdS}_{3}$ with a conical singularity:

$$
S_{\text {ent }}^{c o n}=\frac{c}{3} \ln \left[\frac{\beta_{\text {con }}}{\pi \varepsilon} \sin \left(\frac{2 \pi^{2} \ell}{\beta_{\text {con }}}\right)\right]=\frac{c}{3} \ln \left[\frac{2 \ell^{2}}{r_{+} \varepsilon} \sin \left(\frac{\pi r_{+}}{\ell}\right)\right] .
$$

Equation (45) can be considered as the analytic continuation $r_{+} \rightarrow i r_{+}$of Equation (46) in the next section. The holographic EE of a conical singularity described by a deficit angle $2 \pi\left(1-2 \pi \ell / \beta_{\text {con }}\right)$ is the analytic continuation of the holographic EE for the BTZ black hole with inverse temperature $\beta_{H}=\beta_{\text {con }}$. The analytic continuation corresponds to the exchange of the (compact) timelike with the spacelike direction. This result is a consequence of the modular symmetry (37) of the boundary CFT on the torus relating the BTZ solution and the conical singularity spacetime. In the limit $r_{+} \gg \ell$ the boundary torus corresponding to the BTZ black hole can be approximated by the infinitely long (along the spacelike direction) cylinder $C$. The modular transformation (37) maps the cylinder $C$ into the cylinder $\mathcal{C}$, which has infinitely long direction along the timelike direction and approximates the torus for $\ell \gg r_{+}$. Correspondingly, the EE for the BTZ black hole (46) is transformed into the EE for the conical singularity (45).

An important point, which we have only partially addressed, concerns the role played by the classical solutions of 3D AdS gravity describing conical singularities of the spacetime. Since they represent singular geometries, they cannot be part of the physical spectrum of pure 3D AdS gravity (although they may play a role for gravity interacting with pointlike matter). On the other hand, they are related with the BTZ black hole solutions by modular transformations, and one can associate an EE to them. All this could be very useful for shedding light on the phase transition (analogue to the Hawking-Page transition of four-dimensional gravity $[53,54]$ ), which is expected to take place at $r_{+}=\ell$.

\subsection{Holographic Entanglement Entropy of the BTZ Black Hole}

The spinless BTZ black hole (20) can be considered as the thermalization at temperature $T=T_{H}$ of the AdS spacetime (23). On the 2D boundary of the AdS spacetime, and in the above discussed large temperature limit $r_{+} \gg \ell$, this thermalization corresponds to a plane/cylinder transformation that maps the CFT on the plane $\mathcal{P}$ into the CFT on the cylinder $C$. The conformal map plane/cylinder has the (Euclidean) form given in Equation (30). One can easily check that the above transformation is 
the asymptotic form of the map between the BTZ black hole and $\mathrm{AdS}_{3}$ in Poincare coordinates. The conformal transformation (30) maps the EE of a CFT on the plane $\mathcal{P}$ into the EE of a CFT in the cylinder $C$ [21], i.e. the EE of a CFT at zero temperature in a spacetime with noncompact spacelike dimension into the EE of a CFT at finite temperature. As a result, Equation (6) is transformed into Equation (7) with $\beta=\beta_{H}$. Correspondingly, the holographic EE of the regularized AdS spacetime becomes the holographic EE of the BTZ black hole

$$
S_{\text {ent }}^{B T Z}=S_{\text {ent }}^{C F T}\left(\gamma=2 \pi \ell, \beta=\beta_{H}\right)=\frac{c}{3} \ln \left[\frac{2 \ell^{2}}{\varepsilon r_{+}} \sinh \left(\frac{\pi r_{+}}{\ell}\right)\right] .
$$

The EE (46) still depends on the UV cut-off $\varepsilon$. A renormalized entropy $\tilde{S}_{\text {ent }}^{B T Z}$ can be defined by subtracting the contribution of the vacuum (the zero mass, zero temperature BTZ black hole solution). In terms of the dual CFT, we have to subtract the EE of the zero-temperature vacuum state, which is given by Equation (6) with $\gamma=2 \pi \ell$. The renormalized $\mathrm{EE}$ is therefore given by

$$
\tilde{S}_{\text {ent }}^{B T Z}=S_{\text {ent }}^{B T Z}-S_{e n t}^{v a c}=\frac{\ell}{2 G_{3}} \ln \left[\frac{\ell}{\pi r_{+}} \sinh \left(\frac{\pi r_{+}}{\ell}\right)\right] .
$$

As expected, the renormalized EE vanishes for $r_{+}=0$ (the BTZ black hole ground state).

The holographic EE (47) for the BTZ black hole coincides exactly with the previously derived entropy for the 2D AdS black hole. The 2D AdS black hole is the dimensional reduction of the spinless BTZ black hole. Using the relationship between $2 \mathrm{D}$ and $3 \mathrm{D}$ Newton constant $G_{2}=\ell / 4 G_{3}$, Equation (47) reproduces exactly the result (14).

Macroscopic, i.e. large temperature, black holes (with $r_{+} \gg \ell$ ) correspond, in terms of the 2D CFT, to a thermal wavelength $\beta_{H}$ much smaller than the length $2 \pi \ell$. The expansion of Equation (47) for $r_{+} / \ell \gg 1$ gives

$$
\tilde{S}_{\text {ent }}^{B T Z}=\frac{\pi r_{+}}{2 G_{3}}-\frac{\ell}{2 G_{3}} \ln \frac{\pi r_{+}}{\ell}+\mathcal{O}(1)=S_{B H}-\frac{\ell}{2 G_{3}} \ln S_{B H}+\mathcal{O}(1) .
$$

The leading term in EE is exactly the Bekenstein-Hawking entropy. This leading term describes the extreme situation in which thermal fluctuations dominate completely. In this limit the EE is just a measure of thermodynamic entropy. The EE (the von Neumann entropy) for the CFT becomes extensive and it agrees with the Gibbs entropy of an isolated system of length $\gamma=2 \pi \ell$. The subleading term behaves as $\ln S_{B H}$ and describes the first corrections due to quantum entanglement.

The logarithmic correction in Equation (48) matches exactly the short scale correction for the quantum entropy of a scalar field in the BTZ Euclidean background found in [62] by Mann and Solodukhin. Notice that the authors of [62] consider the case of a single scalar field, therefore we can reproduce their result by using for the central charge $c+\bar{c}=1$. The fact that a 3D bulk calculation of the quantum entropy gives the same result of a boundary CFT calculation is not only a nontrivial check of their correctness, but also a highly nontrivial check of the $\mathrm{AdS}_{3} / \mathrm{CFT}_{2}$ correspondence.

In principle, one could also consider the regime $\beta_{H} \sim 2 \pi \ell$ in which the full quantum nature of the $\mathrm{EE}$ should be evident. However, this regime is singular from the black hole point of view: it corresponds to the $3 \mathrm{D}$ analogous of the Hawking-Page phase transition [53,54]. 
The derivation of the EE for the spinless BTZ black hole can be easily extended to the rotating BTZ solution, obtaining (see [19] for details):

$$
\begin{aligned}
\tilde{S}_{\text {ent }}^{B T Z}= & \frac{\ell}{4 G_{3}} \ln \left\{\frac{\ell^{2}}{\pi^{2}\left(r_{+}+r_{-}\right)\left(r_{+}-r_{-}\right)} \times\right. \\
& \left.\times \sinh \left[\frac{\pi\left(r_{+}+r_{-}\right)}{\ell}\right] \sinh \left[\frac{\pi\left(r_{+}-r_{-}\right)}{\ell}\right]\right\},
\end{aligned}
$$

where $r_{ \pm}$are the positions of the outer and inner horizons. Expanding the previous expression for $r_{+} \gg \ell$ and $r_{+} \gg r_{-}$, we get the same result given in (48).

Let us now briefly comment on the relationship between our approach and that of [5-7,35-37], where the EE of the 2D CFT is computed in terms of 3D bulk geometric quantities (minimal area surfaces). Conversely, in our approach we assume the validity of the EE formulas for the 2D CFT and use them (by means of the AdS/CFT correspondence) for giving a holographic interpretation to the EE of gravitational configurations. The two approaches are complementary in the sense that in [5-7,35-37] bulk gravitational quantities are used to reproduce the EE of the boundary CFT, whereas we use the EE of the CFT to reproduce the EE of bulk gravitational configurations.

\subsection{Entanglement Entropy vs. Thermal Entropy}

In this section we show that the leading term of the holographic EE for the BTZ black hole can be obtained from the large temperature expansion of the partition function of a broad class of 2D CFTs on the torus [19]. This result indicates that black hole EE emerges when the semiclassical notion of spacetime geometry is used to describe the black hole.

In the previous section we have discussed the holographic EE of gravitational configurations in 3D AdS spacetime. In our approach the EE of the boundary CFT, $S_{\text {ent }}^{C F T}(\gamma, \beta)$, is used to probe thermal correlations at scales set by $\beta$ and spatial correlations at scales set by $\gamma$. The bulk description depends crucially on the regime of the $\mathrm{AdS}_{3} / \mathrm{CFT}_{2}$ correspondence we want to investigate.

First of all, we work in the region of validity of the gravity description of the AdS/CFT correspondence, when the AdS length $\ell$ is much larger than the Planck length, which coincides in our units with the 3D Newton constant $G_{3}$ :

$$
\frac{\ell}{G_{3}} \sim c \gg 1
$$

This is the so-called "large $N$ approximation".

Moreover, considering curvature effects much smaller than the curvature $1 / \ell^{2}$ of the AdS spacetime allows the identification of the external parameter $\gamma$ in terms of $\ell$. On the other hand, the thermal scale $\beta$ can be easily identified when a black hole is present in the bulk: $\beta=\beta_{H}=1 / T_{H}$. The semiclassical description for black holes holds when the horizon radius $r_{+}$is much larger than the Planck length, $r_{+} \gg G_{3}$, whereas the holographic EE formula (47) holds for $r_{+} \gg \ell$. We are in the regime where we are allowed to approximate the boundary torus with the cylinder $C$. The path integral of Euclidean quantum gravity on $\mathrm{AdS}_{3}$ is dominated by the contribution coming from the BTZ black hole at $T=T_{H}$. The leading term in the EE (48) describes the main (thermal) contribution of the BTZ geometry and corresponds to the EE for the CFT dominated completely by thermal correlations. When we increase the energy scale, we reach a regime for which contributions coming from geometries different from the 
BTZ instanton cannot be neglected. Quantum entanglement and the subleading term in Equation (48) become relevant.

The other regime we have investigated so far is $\ell \gg r_{+}$, which is related to the previous one by the modular transformation (37). The Euclidean quantum gravity partition function for 3D AdS gravity is now dominated by $\mathrm{AdS}_{3}$ at temperature $T_{H}$. Although the solutions (19) describe singular geometries with conical singularities - therefore they cannot be part of the physical spectrum of the theory-the modular symmetry strongly indicates that they can be used to probe quantum entanglement. In this regime the boundary torus can be described by the cylinder $\mathcal{C}$ and the EE is given by Equation (45).

One may now wonder about the regime $r_{+} \sim \ell$. In this parameter region we cannot approximate the torus with an infinitely long cylinder. $r_{+}=\ell$ is the fixed point of the modular transformations (35), (37) and we have a "large $N$ phase transition", which is the 3D analogue of the Hawking-Page transition [53,54]. Since now the dual boundary CFT lives on the torus $\mathcal{T}\left(\beta_{H}, 2 \pi \ell\right)$, our calculations of the EE on the cylinder lose their validity. Furthermore, it is not a priori evident that the very notion of EE would maintain a sensible physical meaning in a regime where the semiclassical description of gravity is expected to fail.

The most direct way to learn something about the relationship between the two regimes $r_{+} \sim \ell$ and $r_{+} \gg \ell$ is to compare the $\ell \gg \beta$ asymptotic behavior of the thermal entropy $S_{t h}(\beta, \ell)$, derived from the partition function of the dual CFT on the torus, with the EE given by Equation (47). Unfortunately, whereas the EE for a 2D CFT on a cylinder has an universal form, the thermal entropy $S_{t h}(\beta, \ell)$ for the CFT on the torus takes different form depending on the details of the CFT we are dealing with. Let us notice that, despite the intense activity on the subject in the last decade, the exact form of the 2D CFT dual to pure 3D AdS gravity remains still a controversial point [55-57].

Here we will use a simple, albeit not completely general, approach to this problem. We will show that, for the most common 2D CFTs (free bosons, free fermions, minimal models and Wess-Zumino-Witten models), the asymptotic, large temperature $\ell \gg \beta$ behavior of $S_{t h}(\beta, \ell)$ calculated from the partition function of the CFT on the torus reproduces exactly the leading term of the EE (47) for the BTZ black hole.

The partition function of the CFT on the torus, $Z(\tau)$, is a function of the modular parameter $\tau=i \beta / 2 \pi \ell$. Moreover, we will make use of the modular invariance of the partition function under the modular transformation (28) to write $Z(\tau)=Z(-1 / \tau)$. From the partition function one can easily compute the thermal entropy

$$
S_{t h}=\ln Z-\beta \partial_{\beta}(\ln Z) .
$$

We are interested in the asymptotic expansion of $S_{t h}$ in terms of the variable

$$
y=\sinh \left(\frac{2 \pi^{2} \ell}{\beta}\right)
$$

when $y \rightarrow \infty$. The asymptotic form of $S_{t h}(y)$ is determined writing $Z$ as a function of the usual variable $q=\exp (2 \pi i \tau)$. After making use of the modular invariance of the partition function under the modular transformation (28), we then introduce the new variable $\tilde{q}=q(-1 / \tau)=\exp (-2 \pi i / \tau)$ and determine 
the $\tilde{q} \rightarrow 0$ asymptotic expansion of $Z(\tilde{q})$. Finally, we determine $S_{t h}(y)$ by making use of the $y \rightarrow \infty$ asymptotic expansion

$$
\tilde{q}=\frac{1}{4 y^{2}}+\mathcal{O}\left(\frac{1}{y^{4}}\right) .
$$

For free bosons on the torus the asymptotic expansion of the entropy is

$$
S_{t h}(y)=\frac{c}{3} \ln y+\mathcal{O}(\ln (-\ln y)) .
$$

For free fermions on the torus, as well as for minimal models and for Wess-Zumino-Witten models, the entropy has the asymptotic form

$$
S_{t h}=-\frac{c}{6} \ln \tilde{q}+\mathcal{O}(1)=\frac{c}{3} \ln y+\mathcal{O}(1) .
$$

The leading term in the large temperature expansion of the thermal entropy for the four CFT classes on the torus considered in this section is

$$
S_{t h} \sim \frac{c}{3} \ln y=\frac{c}{3} \ln \left(\sinh \frac{2 \pi^{2} \ell}{\beta}\right) .
$$

$S_{t h}$ reproduces, for $\beta=\beta_{H}$, the leading term of the holographic EE for the BTZ black hole given by Equation (47). This result sheds light on the meaning of the holographic EE for the BTZ black hole in particular and, more in general, on the very meaning of entanglement for black holes. In fact, our result indicates that the $\mathrm{EE}$ of a black hole is a semiclassical concept, which has a meaning only for macroscopic black holes in the regime $r_{+} \gg \ell$. Thus, entanglement seems to arise from a purely thermal description of the underlying quantum theory of gravity, which is assumed to describe 3D quantum gravity in the region $r_{+} \sim \ell$. This fact supports the point of view that the microscopic theory describing the BTZ black hole at short scales is unitary. EE is an emergent concept, which comes out when the semiclassical notion of spacetime geometry is used to describe the black hole. The agreement between thermal entropy for the CFT on the torus and holographic EE for the BTZ black hole is limited to the leading term in the large temperature expansion. The subleading terms are not of the same order for the different CFTs we have considered: they behave as $\ln (\ln y)$ for free bosons on the torus, whereas they are $\mathcal{O}(1)$ for the other three cases. Therefore, the subleading terms seem not to be universal (see [63]), but they depend on the actual CFT we are dealing with.

\section{Conclusions}

We have derived, using the AdS/CFT correspondence, simple and general formulas for the entanglement entropy of 2D and 3D AdS black holes. The picture that emerges is intriguing but also not completely surprising. The EE of $2 \mathrm{D}$ and $3 \mathrm{D}$ AdS black holes reduces to the Bekenstein-Hawking entropy - and the subleading terms have the universally predicted logarithmic behavior-only for macroscopic black holes, when thermal correlations dominate. Our result indicates that the EE for a black hole is a semiclassical concept with physical meaning only in the region $\ell \gg \beta$. Away from this regime, the EE codifies information about the full quantum gravity description.

It is important to point out that we have not performed a direct calculation of the EE of matter fields in the BTZ background or other bulk gravitational configurations. In particular, in the 2D and 3D bulk we 
consider pure gravity with no extra matter fields (pure gravity in 3D has no physical propagating degrees of freedom, so we also have no gravitons).

A highly nontrivial check that our procedure gives the correct answer is represented by the exact matching of our results for the logarithmic subleading terms in Equation (48) with those found by Mann and Solodukhin for the EE of scalar fields in the BTZ background [62].

The main drawback of our approach is its limited range of validity. It works only for black holes in AdS spacetime. Moreover, we have only considered the 2D and 3D case; the extension to four-dimensional AdS black holes is in principle possible but computationally more involved.

\section{References and Notes}

1. Vidal, G.; Latorre, J.I.; Rico, E.; Kitaev, A. Entanglement in quantum critical phenomena. Phys. Rev. Lett. 2003, 90, 227902-227905.

2. Kitaev, A.; Preskill, J. Topological entanglement entropy. Phys. Rev. Lett. 2006, 96, 110404-110407.

3. Latorre, J.I.; Lutken, C.A.; Rico, E.; Vidal, G. Fine-grained entanglement loss along renormalization group flows. Phys. Rev. A 2005, 71, 034301-034304.

4. Korepin, V.E. Universality of entropy scaling in one dimensional gapless models. Phys. Rev. Lett. $2004,92,096402-096404$.

5. Ryu, S.; Takayanagi, T. Holographic derivation of entanglement entropy from AdS/CFT. Phys. Rev. Lett. 2006, 96, 181602-181605.

6. Ryu, S.; Takayanagi, T. Aspects of holographic entanglement entropy. JHEP 2006, 0608, 045.

7. Hubeny, V.E; Rangamani, M.; Takayanagi, T. A covariant holographic entanglement entropy proposal. JHEP 2007 , 0707, 062.

8. 't Hooft, G. On The Quantum Structure Of A Black Hole. Nucl. Phys. B 1985, 256, 727-745.

9. Frolov, V.P.; Novikov, I. Dynamical origin of the entropy of a black hole. Phys. Rev. D 1993, 48, 4545-4551.

10. Fiola, T.M.; Preskill, J.; Strominger, A.; Trivedi, S.P. Black hole thermodynamics and information loss in two-dimensions. Phys. Rev. D 1994, 50, 3987-4014.

11. Emparan, R. Black hole entropy as entanglement entropy: A holographic derivation. JHEP 2006, 0606, 012.

12. Hawking, S.; Maldacena, J.M.; Strominger, A. DeSitter entropy, quantum entanglement and AdS/CFT. JHEP 2001, 0105, 001.

13. Bombelli, L.; Koul, R.K.; Lee, J.H.; Sorkin, R.D. A Quantum Source of Entropy for Black Holes. Phys. Rev. D 1986, 34, 373-383.

14. Srednicki, M. Entropy and area. Phys. Rev. Lett. 1993, 71, 666-669.

15. Brustein, R.; Einhorn, M.B.; Yarom, A. Entanglement interpretation of black hole entropy in string theory. JHEP $2006,0601,098$.

16. Maldacena, J.M. Eternal black holes in anti-de-Sitter. JHEP 2003, 0304, 021.

17. Fursaev, D.V. Entanglement entropy in quantum gravity and the Plateau problem. Phys. Rev. D 2008, 77, 124002-124015. 
18. Cadoni, M. Entanglement entropy of two-dimensional anti-de Sitter black holes. Phys. Lett. B 2007, 653, 434-438.

19. Cadoni, M.; Melis, M. Holographic entanglement entropy of the BTZ black hole. Found. Phys. 2010, 40, 638-657.

20. Holzhey, C.; Larsen,F.; Wilczek, F. Geometric and renormalized entropy in conformal field theory. Nucl. Phys. B 1994, 424, 443.

21. Calabrese, P.; Cardy, J.L. Entanglement entropy and quantum field theory. J. Stat. Mech. 2004, 0406, P002.

22. Calabrese, P.; Cardy, J. Entanglement entropy and conformal field theory. J. Phys. A 2009, 42, 504005-504040.

23. Azeyanagi, T.; Nishioka, T.; Takayanagi, T. Near extremal black hole entropy as entanglement entropy via AdS2/CFT1. Phys. Rev. D 2008, 77, 064005-064021.

24. 't Hooft, G. Dimensional reduction in quantum gravity. Salamfest 1993, 284-296, arXiv:gr-qc/9310026.

25. Susskind, L. The world as a hologram. J. Math. Phys. 1995, 36, 6377-6396.

26. Maldacena, J.M. The large N limit of superconformal field theories and supergravity. Adv. Theor. Math. Phys. 1998, 2, 231-252.

27. Susskind, L.; Witten, E. The holographic bound in anti-de Sitter space. High Energy Phys.Theor. 1998, arXiv:hep-th/9805114.

28. Peet, A.W.; Polchinski, J. UV/IR relations in AdS dynamics. Phys. Rev. D 1999, 59, 065011-065015.

29. Polchinski, J.; Susskind, L.; Toumbas, N. Negative energy, superluminosity and holography. Phys. Rev. D 1999 , 60, 084006-084013.

30. Solodukhin, S.N. Entanglement entropy of black holes and AdS/CFT correspondence. Phys. Rev. Lett. 2006, 97, 201601-201604.

31. Fursaev, D.V. Entanglement entropy in critical phenomena and analogue models of quantum gravity. Phys. Rev. D 2006, 73, 124025-124036.

32. Frolov, V.P.; Fursaev, D.V.; Zelnikov, A.I. Statistical origin of black hole entropy in induced gravity. Nucl. Phys. B 1997, 486, 339-352.

33. Frolov, V.P.; Fursaev, D.V. Mechanism of generation of black hole entropy in Sakharov's induced gravity. Phys. Rev. D 1997, 56, 2212-2225.

34. Aharony, O.; Gubser, S.S.; Maldacena, J.M.; Ooguri, H.; Oz, Y. Large N field theories, string theory and gravity. Phys. Rept. 2000, 323, 183-386.

35. Fursaev, D.V. Proof of the holographic formula for entanglement entropy. JHEP 2006, 0609, 018.

36. Michalogiorgakis, G. Entanglement entropy of two dimensional systems and holography. JHEP $2008,0812,068$.

37. Sun, J.R. Note on Chern-Simons term correction to holographic entanglement entropy. JHEP 2009, 0905, 061.

38. Cadoni, M.; Mignemi, S. Nonsingular four-dimensional black holes and the Jackiw-Teitelboim theory. Phys. Rev. D 1995, 51, 4319-4329. 
39. Cadoni, M.; Mignemi, S. Entropy of 2D black holes from counting microstates. Phys. Rev. D 1999, 59, 081501-081506.

40. Cadoni, M.; Mignemi, S. Asymptotic symmetries of $\operatorname{AdS}(2)$ and conformal group in d $=1$. Nucl. Phys. B 1999, 557, 165-180.

41. Cadoni, M.; Cavaglia, M. Open strings, 2D gravity and AdS/CFT correspondence. Phys. Rev. D 2001, 63, 084024-084035.

42. Cadoni, M.; Cavaglia, M. Two-dimensional black holes as open strings: A new realization of the AdS/CFT duality. Phys. Lett. B 2001, 499, 315-320.

43. Susskind, L.; Uglum, J. Black hole entropy in canonical quantum gravity and superstring theory. Phys. Rev. D 1994, 50, 2700-2711.

44. Fursaev, D.V. Temperature and entropy of a quantum black hole and conformal anomaly. Phys. Rev. D 1995, 51, 5352-5355.

45. Mann, R.B.; Solodukhin, S.N. Universality of quantum entropy for extreme black holes. Nucl. Phys. B 1998, 523, 293-307.

46. Kaul, R.K.; Majumdar, P. Logarithmic correction to the Bekenstein-Hawking entropy. Phys. Rev. Lett. 2000, 84, 5255-5257.

47. Carlip, S. Logarithmic corrections to black hole entropy from the Cardy formula. Class. Quant. Grav. 2000, 17, 4175-4186.

48. Ghosh, A.; Mitra, P. Entropy in dilatonic black hole background. Phys. Rev. Lett. 1994, 73, 2521-2523.

49. Mukherji, S.; Pal, S.S. Logarithmic corrections to black hole entropy and AdS/CFT correspondence. JHEP 2002, 0205, 026.

50. Setare, M.R. Logarithmic correction to the brane equation in topological Reissner-Nordstroem de Sitter space. Phys. Lett. B 2003, 573, 173-180.

51. Medved, A.J.M. A comment on black hole entropy or why Nature abhors a logarithm. Class. Quant. Grav. 2005, 22, 133-142.

52. Domagala, M.; Lewandowski, J. Black hole entropy from quantum geometry. Class. Quant. Grav. 2004, 21, 5233-5244.

53. Hawking, S.W.; Page, D.N. Thermodynamics of black holes in anti-de Sitter space. Commun. Math. Phys. 1983, 87, 577-588.

54. Kurita, Y.; Sakagami, M.A. CFT description of three-dimensional Hawking-Page transition. Prog. Theor. Phys. 2005, 113, 1193.

55. Carlip, S. Conformal field theory, (2+1)-dimensional gravity, and the BTZ black hole. Class. Quant. Grav. 2005, 22, R85-R124.

56. Witten, E. Three-dimensional gravity revisited. High Energy Phys. Theor. 2007, arXiv:0706.3359 [hep-th].

57. Maloney, A.; Witten, E. Quantum gravity partition functions in three dimensions. JHEP 2010, $1002,029$.

58. Brown, J.D.; Henneaux, M. Central charges in the canonical realization of asymptotic symmetries: An example from three-dimensional gravity. Commun. Math. Phys. 1986, 104, 207-226. 
59. Banados, M.; Teitelboim, C.; Zanelli, J. The black hole in three-dimensional space-time. Phys. Rev. Lett. 1992, 69, 1849-1851.

60. Banados, M.; Henneaux, M.; Teitelboim, C.; Zanelli, J. Geometry of the (2+1) black hole. Phys. Rev. D 1993, 48, 1506.

61. Carlip, S.; Teitelboim, C. Aspects of black hole quantum mechanics and thermodynamics in (2+1)-dimensions. Phys. Rev. D 1995, 51, 622-631.

62. Mann, R.B.; Solodukhin, S.N. Quantum scalar field on three-dimensional (BTZ) black hole instanton: Heat kernel, effective action and thermodynamics. Phys. Rev. D 1997, 55, 3622-3632.

63. Page, D.N. Hawking radiation and black hole thermodynamics. New J. Phys. 2005, 7, 203.

(C) 2010 by the authors; licensee MDPI, Basel, Switzerland. This article is an open access article distributed under the terms and conditions of the Creative Commons Attribution license (http://creativecommons.org/licenses/by/3.0/.) 